\title{
Addressing the issue of fog and haze: A promising perspective from meteorological science and technology
}

Fog and haze are two kinds of weather phenomena. Fog is referred to as water droplets suspended in the air near earth's surface, which reduces the visibility to less than $1 \mathrm{~km}$. A fog consisting of ice crystals is called ice fog. Haze is the particles suspended in the air that reduce visibility by scattering the light, and is usually a mixture of aerosol and photochemical smog. Fog and haze usually coexist. The occurrence and evolution of haze and fog are closely related with meteorological conditions.

In the recent decades, due to the rapid economic growth and climate change, haze and fog frequently occurred in China have become more intense, harming human health, affecting people's daily life, and threatening the sustainable development of economy and society. How to mitigate and control haze and fog are very important issues with widespread concerns by the public and government at all levels. In this special topic, four articles are selected on the research of the severe haze and fog event over eastern China in January 2013. The strategies and measures on the haze and fog in the meteorological perspective are proposed.

In January 2013 a severe fog and haze event of strong intensity, long duration, and extensive coverage occurred over eastern China (Zhang et al., 2014). Accompanied with this event was heavy air pollution for 27 days (i.e., $87 \%$ of the entire month) in Beijing-Tianjin-Hebei region (Wang Z F et al., 2014). The highest hourly concentration of $\mathrm{PM}_{2.5}$ in Beijing once reached $680 \mu \mathrm{g} \mathrm{m}^{-3}$ (Wang Y S et al., 2014). Because of the long-lasting, extremely heavy air pollution in Beijing, Beijing Meteorological Observatory issued an orange haze alert for the first time in history (Ding and Liu, 2014).

\section{Impact of meteorological conditions on the haze and fog event}

In January 2013, both the atmospheric background fields and daily evolution of meteorological factors had major impacts on the severe haze and fog event (Zhang et al., 2014). The atmospheric background fields in January 2013 included a weaker East Asian winter monsoon, reduced horizontal wind velocity and vertical wind shear, and increased tropospheric air temperature and inversion in lower troposphere over eastern China, which are favorable for the occurrence and persistence of the heavy haze and fog event. The daily evolution of the haze and fog event is affected by the atmospheric dynamical factors (vertical horizontal wind shear in middle and lower troposphere and surface wind velocity) and thermodynamic factors (stratification instability in lower troposphere, near-surface inversion, and near-surface dewpoint deficit). These meteorological factors explain over two-thirds of the variance of the daily fog and haze evolution.

The transfer of the gaseous state to the particulate, which depends on meteorological conditions, plays an important role in this event (Wang Y S et al., 2014). The rapid transfer is the internal triggering factor for the onset and persistence of the haze pollution in January 2013. The heterogeneous reaction of gaseous pollutants on the surface of fine particles changes the size and chemical composition of the particles, and increases the proportion of secondary inorganic aerosols, which strengthen the hygroscopicity of particles and promote the formation of the heavy haze pollution.

The regional atmospheric transport influenced the distribution of the $\mathrm{PM}_{2.5}$ concentration in January 2013 over Beijing-Tianjin-Hebei region, which is demonstrated by the results of numerical simulation (Wang Z F et al., 2014). In typical

Citation: Mu M, Zhang R H. 2014. Addressing the issue of fog and haze: A promising perspective from meteorological science and technology. Science China: Earth Sciences, 57: 1-2, doi: 10.1007/s11430-013-4791-2 
cities (e.g., Beijing, Tianjin, Qinhuangdao, and Cangzhou), the contributions of the $\mathrm{PM}_{2.5}$ concentration from the cross-border transport outside Beijing-Tianjin-Hebei region and the intra-regional transport are equivalent to the contribution from the local pollution sources. In addition, the interaction between meteorological conditions and air pollution has an important effect on the formation of the heavy haze.

In China the long-term trend of haze days in the past 50 years increases significantly, whereas fog days have decreased since 1990 (Ding and Liu, 2014). The global warming increases air temperature and saturation specific humidity, leading to the decrease of near-surface relative humidity, which may have an impact on the environmental conditions for the formation of haze and fog. The declined relative humidity for haze days implies the dampened transformation of haze particles to fog droplets, which may be the main cause for the decrease of fog days.

\section{Strategies in addressing the haze and fog event from meteorological aspect}

(1) Haze and fog forecasting. An accurate forecast of haze and fog is an important prerequisite in addressing their harm. The occurrence and maintenance of haze and fog depend largely on meteorological conditions. For example, more than $2 / 3$ variance of the daily haze and fog evolution in January 2013 can be explained by meteorological factors (Zhang et al., 2014). So far, the technology of the short-term weather forecast within 3 days is well developed. The long-term weather prediction is also of important reference. Therefore, there is a clear feasibility in carrying out the forecast of haze and fog in line with meteorological factors.

(2) Controlling air pollutant emission based on meteorological conditions. The emission of pollutants into the air is undoubtedly the prerequisite for air pollution. However, the region and intensity of air pollution occurrence are closely related to meteorological conditions. In this context, the control of pollutant emission can be adopted based on meteorological conditions. A scientific policy-making in the air pollution control will be beneficial to the cost reduction and efficiency enhancement.

(3) Inter-regional treatment on haze pollution. The air flow can result in the transportation of pollutants among different regions, and it plays a crucial role in the distribution of pollutants. For example, the results of numerical simulation on the fog and haze event in January 2013 (Wang Z F et al., 2014) show that the inter-regional transport of haze is a main factor affecting regional haze pollution, indicating the importance of inter-regional cooperation in air pollution control and prevention. Therefore, choosing cooperation regions according to the actual meteorological conditions is effective measures to reduce haze effects and to avoid the excessive and blind control.

(4) Improving haze and fog observations. Although the haze and fog is routinely observed in meteorological stations, big problems still exist in haze and fog observations. Because fog and haze are frequently mixed together, they can be hardly distinguished by operational observation methods at present. Therefore, the acquirement of accurate and reliable fog and haze data through improving the equipment for fog and haze observations and establishing the observing network of high spatial and temporal resolutions are fundamental for addressing the fog and haze issues.

In summary, meteorological science and technology can play important roles in observation, formation mechanism, numerical simulation, forecast, prevention, and control of haze and fog events. In addressing the fog and haze issues, the close cooperation is needed among scientists and technicians from environmental, meteorological, and industrial sectors. There is a long way ahead. We hope that the papers on this special topic can promote the research and the cooperation in China on addressing the fog and haze issues.

Ding Y H, Liu Y J. 2014. Analysis of long-term variations of fog and haze in China in recent 50 years and their relations with atmospheric humidity. Sci China Earth Sci, 57: 36-46

Wang Y S, Yao L, Wang L L, et al. 2014. Mechanism for the formation of the January 2013 heavy haze pollution episode over central and eastern China. Sci China Earth Sci, 57: 14-25

Wang Z F, Li J, Wang Z, et al. 2014. Modeling study of regional severe hazes over mid-eastern China in January 2013 and its implications on pollution prevention and control. Sci China Earth Sci, 57: 3-13

Zhang R H, Li Q, Zhang R N. 2014. Meteorological conditions for the persistent severe fog and haze event over eastern China in January 2013. Sci China Earth Sci, 57: 26-35

MU Mu

Key Laboratory of Ocean Circulation and Waves, Institute of Oceanology, Chinese Academy of Sciences E-mail: mumu@qdio.ac.cn

ZHANG RenHe State Key Laboratory of Severe Weather, Chinese Academy of Meteorological Sciences E-mail: renhe@cams.cma.gov.cn 